\title{
A DECOMPOSITION INTO HOMEOMORPHIC HANDLEBODIES WITH NATURALLY EQUIVALENT INVOLUTIONS
}

ROGER B. NELSON

\author{
Department of Mathematical Sciences \\ Ball State University \\ Muncie, IN 47306
}

(Peceived September 12,1988 )

\begin{abstract}
Downing [6] extended the well-known result that any closed 3-manifold $x$ contains a handlebody $H$ such that $c l(X-H)$ is homeomorphic to $H$ in the case where $X$ is a compact 3-manifold with nonvoid boundary. We show that if $X$ is a compact 3-manifold with involution $h$ having 2 -dimensional fixed point set, then $X$ contains an h-invariant handlebody $H$ such that the involutions induced on $H$ and $c l(X-I I)$ are naturally equivalent.
\end{abstract}

KEY WORDS AND PHARSES. 3-manifold, involution, handlebody, equivalent involutions. 1980 AMS SUBJECT CLASSIFICATION CODES. Primary 57Q15, 57S17, 57S25.

1. INTRODUCTION.

In this paper all spaces and maps are plecewise linear ( $P L$ ) in the sense of Rourke and Sanderson [1]. Thus all spaces can be triangulated and all maps and subspaces are PL. This is no restriction as every 3-manifold has a triangulation by Bing [2]. Notation follows Nelson [3], [4], and Rourke and Sanderson [1].

Let $X$ be a closed 3-manifold with triangulation $T$. If $S$ is the 1-skeleton of $T$ and $\mathrm{H}_{1}$ is a regular neighborhood of $\mathrm{s}$, then it is a classical result, see seifert and Threlfa]l [5, p. 219], that $\mathrm{H}_{1}$ and $\mathrm{H}_{2}=\mathrm{cl}(\mathrm{X}-\mathrm{H})$ are homeomorphic handlebodies. Downing [6] extended this result, showing that every 3-manifold with nonvoid boundary also contains a handlebody which is homeonorphic to its complement. It is the purpose of this paper to show that these results can be further extended so that certain symmetries are respected. These symmetries are those realized as involutions of $X$ with orientable 2-dimensional fixed point sets.

Recall that an involution of $Y$ is a homeomorphism on $X$ of period two. Involutions $h_{1}: x_{i}+x_{1}, i=1,2$, are equivalent, denoted $h_{1} \sim h_{2}$, if there exists a homeomorphism $f: x_{1}+x_{2}$ such that $h_{1}=r^{-1} \circ h_{2} \circ f$. The fixed point set of involution $h: X+X$ is $F(h)=\{x \in X \mid h(x)=x\}$. We shall prove the following: 
THEOREM 1. Let $\mathrm{X}$ be an orientable 3-manifold and $\mathrm{h}: \mathrm{X} \rightarrow \mathrm{X}$ an involution with 2dimensional, orientable fixed point set $F$. Then there exist complementary, hinvariant handlebodies $\mathrm{H}_{1}$ and $\mathrm{H}_{2}$ in $\mathrm{X}$ such that $\mathrm{h} \mid \mathrm{H}_{1} \sim \mathrm{h} / \mathrm{H}_{2}$ ・

THEOREM 2. Let $\mathrm{f}: \mathrm{H}_{1}+\mathrm{H}_{2}$ be the homeomorphism such that $\mathrm{h} / \mathrm{H}_{1}=\mathrm{f}^{-1} \circ\left(\mathrm{h} / \mathrm{H}_{2}\right) \circ \mathrm{f}$ thereby establishing the equivalence $h\left|H_{1} \sim h\right| H_{2}$ of Theorem 1 . Then $f \mid \partial H_{1}$ may be assumed to be the identity on $\partial \mathrm{H}_{1}=\mathrm{H}_{1} \cap \mathrm{H}_{2}=\partial \mathrm{H}_{2}$.

By Nelson [4; Thm. C] the involutions $h / H_{1}$ and $h / H_{2}$ are equivalent if and only if (i) their fixed point sets are homeomorphic and (ii) either both fixed point sets separate or both fall to separate. In Nelson [3; 1emma 2.1] it was shown that a 2dimensional fixed point set of an involution on an orientable handlebody separates the handlebody if and only if the l-dimenstonal fixed point set of the involution induced on the boundary separates the boundary. Since $H_{1}$ and $H_{2}$ share a common boundary, we need only show that $H_{1}$ can be chosen so that $H_{1} \cap F \simeq H_{2} \cap F$. Hence, Theorem follows immediately from the following lemma:

LEMMA 1. Let $X$ be a compact 3-manifold and $h: x \rightarrow X$ an involution with 2dimensional, orientable fixed point set $F$. Then there exist complementary, hinvariant handlebodies $\mathrm{H}_{1}$ and $\mathrm{H}_{2}$ in $X$ such that $\mathrm{H}_{1} \cap \mathrm{F} \approx \mathrm{H}_{2} \cap \mathrm{F}$.

The 3-manifold $X$ may be either orientable or nonorientable in this lemma. $X$ mast be assumed to be orientable in Theorem 1 in order to invoke Nelson [4; Thm. C]. Section 2 contains the proof of this lemma in the case when $X$ is a closed manifold. The proof is modified in section 3 to cover the case when $X$ has nonvold boundary. The proof of Theorem 2 is provided in section 4. Both sections 3 and 4 are modifications of proofs which appear elsewhere. These sections refer heavily to the proofs which they modify.

2. X IS CLOSED.

Our approach is to pick an initial handlebody $H_{1}$ that will be repeatedly modified until $H_{1} \cap F_{1} \approx H_{2} \cap F_{1}$ for each component $F_{i}$ of $F$. This identity is established by showing $\mathrm{H}_{1} \cap \mathrm{F}_{i}^{+} \simeq \mathrm{H}_{2} \cap \overline{\mathrm{F}_{i}^{-}}$and $\mathrm{H}_{1} \cap \overline{\mathrm{F}_{i}^{-}} \simeq \mathrm{H}_{2} \cap \mathrm{F}_{i}^{+}$, where $\mathrm{F}_{i}^{+}$and $\mathrm{F}_{i}^{-}$are "homeomorphic halves" of $\mathrm{F}_{1}$.

The Initial $\mathrm{H}_{1}$ : We note first that any component $F_{i}$ of $F$ is a closed, orientable surface. Any such surface admits an involution $g_{i}$ with a separating fixed point set consisting of at most two simple closed curves. This separates $F_{1}$ into $F_{i}^{-}$and $F_{1}^{+}$ where $g_{i}\left(F_{i}^{-}\right)=F_{i}^{+}$.

Let $X_{h}^{*}$ be the orbit space of $h$. Since $F$ is 2-dimensional, $X_{h}^{*}$ is a bordered 3manifold with $\partial X_{h}^{*} \simeq F$. Triangulate $\underset{h}{\partial}$ so that the triangulation of component $F_{i}$ is $g_{i}$-invariant. Since $\underset{h}{\partial x_{h}^{*}} 1$ collared, Rouke and Sanderson $[1 ;$ Cor. 2.26], the triangulation of $\partial X_{h}^{*}$ can be extended to a triangulation $T^{*}$ of $X_{h}^{*}$. Let $T$ be the lift of $T^{*}$ to $X$. Then $T$ is an h-invariant triangulation of pair $(X, F)$ such that $F_{1}^{+}$is triangulated identically to $\mathrm{F}_{i}^{-}$for each 1 . 
Denote by $T^{(n)}$ the $n$th derived subdivision of $T$ and by $S^{(n)}$ the collection of all edges and vertices of $T^{(n)}$ lying on the 1-skeleton $S$ of $T$. Let $H_{1}=N\left(S^{(2)}, T^{(2)}\right)$ and $\mathrm{H}_{2}=\left(\mathrm{X}-\mathrm{H}_{1}\right), \mathrm{H}_{1}$ and $\mathrm{H}_{2}$ are homeomorphic orientable handlebodies. Since $\mathrm{T}$ triangulates $F, H_{1} \cap F_{i}$ is the connected, orientable surface $F_{i}$ minus $k$ disks, where $k$ is the number of 2-simplices in the triangulation of $F_{1} \cdot H_{2} \cap F_{1}$ consists of $k$ disks.

Modification Procedures: Two procedures are used to repeatedly modify $T$ near each $\mathrm{F}_{i}$. The result of modification by either procedure is that the modified handlebody $\tilde{\mathrm{H}}_{1}$ is isomorphic to $\tilde{\mathrm{H}}_{1}$ plus a 3-handle of index one and the new complementary handlebody $\tilde{\mathrm{H}}_{2}$ is isomorphic to $\mathrm{H}_{2}$ plus a 3-handle of index one. Hence, after each modification we are again left with homeomorphic, complementary handlebodies.

The 3-simplices of $T$ are in h-invariant pairs. We say such a pair is near $F_{i}$ if the two 3-simplices of the pair intersect in $F_{i}$. The procedures below correspond to the cases where 3-simplices intersect in an edge or 2-face in $F_{i}$. We will not need to employ modifications exploiting simplices intersecting $F_{i}$ in a vertex.

Procedure $\alpha$ : The net result of this procedure is to renove one edge from $T \cap F_{i}$. Intuitively, $\tilde{\mathrm{H}}_{1} \cap \mathrm{F}_{i}$ contains "less" of $\mathrm{F}_{i}$ than $\mathrm{H}_{1} \cap \mathrm{F}_{i}$.

Let $e$ be any edge of $T$ lying in $F_{1}$. Then $e$ is the common edge of two 3simplices, $s_{1}$ and $s_{2}$, such that $h\left(s_{1}\right)=s_{2}$ and $s_{1} \cap s_{2}=\{e\}$. Let $e_{1}$ and $e_{2}$ be the edges of $\mathrm{T}^{(1)}$ connecting the vertices of $e$ with the barycenter of $s_{1}$. Modify $s^{(2)}$ to get $\hat{S}^{(2)}$ by deleting from $\mathrm{S}^{(2)}$ all vertices and edges of $\mathrm{T}^{(2)}$ lying on $\mathrm{e}$ and adding to $\mathrm{s}^{(2)}$ all vertices and edges of $\mathrm{T}^{(2)}$ lying on $\left(\mathrm{e}_{1} \cup \mathrm{e}_{2}\right) \cup \mathrm{h}\left(\mathrm{e}_{1} \cup \mathrm{e}_{2}\right)$. We replace $\mathrm{H}_{1}$ by $\tilde{\mathrm{H}}_{1}=\mathrm{N}\left(\hat{\mathrm{S}}^{(2)}, \mathrm{T}^{(2)}\right)$. $\tilde{\mathrm{H}}_{1}$ is homeomorphic to $\mathrm{H}_{1}$ plus a 3 -handle of index one. $\widetilde{\mathrm{H}}_{2}=\mathrm{cl}\left(\mathrm{X}-\tilde{\mathrm{H}}_{1}\right)$ is homeomorphic to $\mathrm{H}_{2}$ plus a 3-handle of index one, the cocore of which lies in a disk bounded by $\left(e_{1} \cup e_{2}\right) \cup h\left(e_{1} \cup e_{2}\right)$.

Procedure $\beta$ : The net result of this procedure is to add an h-invariant edge to $T$ which intersects $F_{1}$ in a point. As a result, $\tilde{H}_{1} \cap F_{i}$ contains a disk component not in $\mathrm{H}_{1} \cap \mathrm{F}_{1}$.

Let $a_{1} a_{2} a_{3}$ be any 2-simplex of $T$ in $F$. Then $a_{1} a_{2} a_{3}$ is the common $f$ ace of 3-simplices $a_{1} a_{2} a_{3} a_{4}$ and $a_{1} a_{2} a_{3} a_{5}$ such that $h\left(a_{4}\right)=a_{5}$. The union of these simplices is a double tetrahedron we shall retriangulate. The new triangulation consists of the simplices $a_{1} a_{2} a_{4} a_{5}, a_{1} a_{3} a_{4} a_{5}, a_{2} a_{3} a_{4} a_{5}$ and their faces. It is easily checked that this new triangulation is h-invariant. Let $\tilde{T}$ be the triangulation of $x$ obtained by replacing the former simplices of the double tetrahedron by the new ones. Substitute for $\mathrm{H}_{1}, \tilde{\mathrm{H}}_{1}=\left|\mathrm{N}\left(\widetilde{\mathrm{S}}^{(2)}, \widetilde{\mathrm{T}}^{(2)}\right)\right|$. $\tilde{\mathrm{H}}_{1}$ is homeomorphic to $\mathrm{H}_{1}$ plus a 3 -handle whose core is the edge $a_{4} a_{5} \cdot \tilde{H}_{2}=c 1\left(x-H_{2}\right)$ is also homeomorphic to $H_{1}$ plus a 3-handle of index one. Employing the Modifications: The $\alpha$ and $\beta$ modifications are repeated independently near each $F_{i}$. Let $f$ be the number of 2-simplices and $p$ the number of vertices in the triangulation of $\mathrm{F}_{i}^{+}$(and also of $\overline{F_{i}^{-}}$). We assume that the original $T$ was chosen so that $p<2 f$ for each fixed point component $F_{i}$. 
CASE 1. $p>f$. First apply $B \mathrm{p}-\mathrm{f}$ time; where $\mathrm{a}_{1} \mathrm{a}_{2} \mathrm{a}_{3} \in \mathrm{F}_{i}^{-}$. In order to carry this out we mist have that $p-f \leqslant f$. Bit this is equivalent to the statement that $\chi\left(\mathrm{F}_{i}^{-}\right)<3 \mathrm{f}-\mathrm{e}$ which is inmediate when $\chi\left(\mathrm{F}_{i}^{-}\right)=\frac{1}{2} \chi\left(\mathrm{F}_{i}\right)<0$ and easily checked in the remaining case when $F_{i} \simeq s^{2}$. Next, apply procedure $\alpha$ to every edge in $F_{i}^{-}$. Then both $\mathrm{H}_{1} \cap \mathrm{F}$ and $\mathrm{H}_{2} \cap \mathrm{F}$ consist of $\mathrm{p}$ disk components and one component homeomorphic to $\mathrm{F}^{+}$(or $\mathrm{F}^{-}$) mi nus $\mathrm{p}$ disks.

CASE 2. $f>p$. First apply procedure $\beta f-p$ times to sinplices in $F_{i}^{+}$. Then use procedure $\alpha$ to remove all edges of $\mathrm{T}$ from $\mathrm{r}_{i}^{+}$. The result is that both $H_{l} \cap \mathrm{F}$ and $\mathrm{H}_{2} \cap \mathrm{F}$ consist of $\mathrm{f}$ disk components and one component homeomorphic to $\mathrm{F}^{+}$(or $\mathrm{F}^{-}$) less $\mathrm{f}$ disks.

3. $\mathrm{Y}$ IS NONVOID.

In this section we nake the adjustments necessary in the above proof for the case $\partial X \neq \phi$. Notice that only the intial choice of $H_{1}$ was dependent upon $x$ having empty boundary. The modification procedures do not depend upon assumptions about $\partial x$.

First, it was noted that $F_{i}$ is a closed, orientable surface. If $\partial x \neq \phi$, this is no longer necessarily true. However, $F_{i}$ will be a closed orientable surface less a collection of disks. Such a surface also admits an involution $g_{1}$ with a separating fixed point set consisting of simple closed curves and arcs.

No longer is it true that a regular neighborhood of the l-skeleton of triangulation of $X$ will have a homeonorphic complement in $x$. Rather, we must show that Downing's construction of the handlebody $H_{1}$ can be carried out equivariantly.

Let $B_{i}, i=1, \ldots, n$ be the boundary components of $X$ and let $X^{\prime}=X U_{k}\left(U M_{i}\right)$, where $M_{i}$ is the handlebody with $\partial M \simeq B_{i}$ and $k / B_{i}$ mapping $B_{i}$ homeonorphically to $\partial M_{i} \cdot$ $X^{\prime}$ is a closed 3 -manifold and accurdingly has a classical decomposition into homeomorphic handlebodies $H_{1}^{\prime}$ and $H_{2}^{\prime}=c 1\left(X^{\prime}-H_{1}^{\prime}\right)$. By Down Ing [6; Lemma 1], each $M_{i}$ is isotoped by $G$ in $X^{\prime}$ onto $N_{i}$ where $N_{i j}=N_{i} \cap H_{j}^{\prime}, j=1,2$, are specially positioned in $\mathrm{H}_{1}$ and $\mathrm{H}_{2} \cdot\left(\mathrm{M}_{\mathrm{i}}\right.$ and $\mathrm{N}_{i}$ are, respectively, just regular neighborhoods of the l-dimensional sets denoted $Y$ and $\mathrm{Y}^{1}$ in Downing's proof.) It is then shown that $X \simeq X^{\prime}-\underset{i}{U} M_{i}$ and that $H_{1}^{\prime}=H_{1}-\underset{i}{\prime} N_{i 1}$ and $H_{2}^{\prime}=H_{2}-\underset{i}{U} N_{i 2}$ are homeomorphic handlebodies with $\mathrm{H}_{2}=\mathrm{cl}\left(\left(\mathrm{X}^{\prime}-\mathrm{UN}_{\mathrm{i}}\right)-\mathrm{II}_{1}\right)$.

The isotopy $G$ of $X^{\prime}$ moving each $M_{i}$ to $N_{i}$ yields a homeomorphism $f: X^{\prime} \rightarrow X^{\prime}$ such that $f\left(N_{i}\right)=M_{i}$ for all $i . f\left(H_{1}\right)$ and $f\left(H_{2}\right)$ are homeomorphic handlebodies such that $f\left(H_{2}\right)=c l\left(x-f\left(H_{1}\right)\right)$. We do not yet know, however, that $f\left(H_{1}\right)$ and $f\left(H_{2}\right)$ are $h-$ invariant. $H_{1}^{\prime}$ and $H_{2}^{\prime}$ may, however, be assumed h-invariant as in section 2 . But if (i) $h$ can be extended to an involıtion $h^{\prime}: X^{\prime} \rightarrow X^{\prime}$ and (ii) if the isotopy $G$ can be constructed to commute with $h^{\prime}$, then $f\left(H_{1}\right)$ and $f\left(H_{2}\right)$ will be $h$-invariant as needed.

To extend $h$ to $h$ ' we need only kuow that any involution (free or with 1dimensional fixed point set) on ${ }^{1 M_{1}}$ is equivalent by $k_{1}=k \mid B_{i}$ to the restriction to $B_{i}$ of an involution on $M_{i}$. The'n attaching $M_{i}$ to $x$ by $k_{i}$ allows $h$ to be extended into $M_{i}$. Let $M_{i}=A \times I$ where $A$ is a 2-spher minus disks. If $h$ is a free involution 
of $\partial M_{i}$, then $h \mid M_{i}$ is equivalent to $\ell$ given $\ell(m, t)=(\alpha(m), t)$ where $\alpha$ is the free involution on $A$ derived from the antipodal map on $\mathrm{s}^{2}$ by removing invariant pairs of disks.

If $h / \partial M_{1}$ has a l-dimensional fixed point set, it will consist only of simple closed curves. In this case $h / M_{i}$ is equivalent to $l$ where $\alpha$ is an involution of $A$ with arcs as fixed point components. If the fixed point. set separates, than $\alpha$ may be derived from the reflection in a great circle of $\mathrm{s}^{2}$ by removing invariant disks from the fixed point circle and invariant pairs of disks disjoint from the circle of fixed points.

Suppose $h / M_{i}$ has a nonseparating fixed point set consisting of $k$ simple closed curves. Let $M_{i}=A \times I$ where $A$ is $S^{1} \times S^{1}$ minus disks. The involution $m: S^{l} \times S+s^{l} \times S^{l}$ given by $\gamma\left(s_{1}, s_{2}\right)=\left(s_{2}, s_{1}\right)$ has one nonseparating fixed point loop. Again, $h / M_{i} \sim \alpha$ where $\alpha$ is derlved from $\gamma$ by removing $k$ invariant disks from the nonseparating fixed point and an appropriate number of invariant pairs of disks disjoint from the fixed point set.

Let $A \times[-1,1]$ be embedded in $X^{\prime}$ by $j$. such that $h^{\prime} \mid j(A \times[-1,1])$ preserves the locally defined product structure. Using an invariant collar $C$ on $B_{i}$ in $X$, $j$ can be extended to an embedding, also called $j$, of $A \times[-1,3]$ into $X$ ', such that

$j(A \times\{1\})=B_{i}$ and so that $h^{\prime} \mid f(A \times[-1,3])$ is fiber preserving. By Kim and Tollefson [7; Thm. B], $h^{\prime} \mid C \sim \ell$ where $\ell: B_{1} x[-1,3]+B_{1} \times[-1,3]$ is given by $\ell(b, t)=\left(\left(h^{\prime} \mid B_{f}\right)(b), t\right)$. Since $j(A \times\{1\})$ is an invariant subspace of $B_{i}, j(A \times[1,3])$ is an invariant subspace of $C$ with $h^{\prime} \mid j(A \times[1,3]) f i b e r$ preserving. Replacing $T \times[-1,3]$ in the proof of Downing [6; lemma 1] by $A \times[-1,3]$, the isotopy $G_{t}$, with support in $j(A \times[-1,3])$, taking $M_{1}$ to $N_{1}$ may be ass umed to commute with $h^{\prime}$. That is, we may assume that $G_{t}$ preserves the fiber structure on $f(A \times[-1,3])$.

In the case where the boundary component $B_{i}$ is not h-invariant, such care in choosing the isotopy $G_{t}$ is unnecessary. Any isotopy $G_{t}$, with support in a small neighborhood of $M_{i}$, will suffice as long as $h^{\prime} G_{t}$ is applied near $M_{j}=h M_{i}$.

4. PROOF OF THEOREM 2.

In this section we show that the equivalence $h\left|H_{1} \sim h\right| H_{2}$ is in some sense natural. That is, we show that the homeomorphism $\mathrm{f}: \mathrm{H}_{1}+\mathrm{H}_{2}$ establishing this equivalence may be assumed to be the identity on $\partial \mathrm{H}_{1}=\mathrm{H}_{1} \cap \mathrm{H}_{2}=\partial \mathrm{H}_{2}$. This we do by modifying the proofs of Nelson [4, Theorem B] and Nelson [3, Theorem 4.1].

The proof of the equivalence in the above referenced papers is by induction on the common genus of the handlebodies $\mathrm{H}_{1}$ and $\mathrm{H}_{2}$. Nelson [4; Lemma 2.1] provides $\left(h \mid H_{i}\right)$-invarlant, nonseparating, properly embedded disks $D_{i} c H_{i}$ such that cutting along the $D_{i}$ lowers the genera of the fixed point sets $F\left(h \mid H_{i}\right), i=1,2$. Let $H_{i}^{\prime}=H_{i}-N\left(D_{i}\right), i=1,2$, be the handlebodies resulting from cutting along $D_{i}$. By the induction hypothesis, $h\left|H_{i}^{\prime} \sim h\right| H_{2}^{\prime}$ and also $h\left|N\left(D_{1}\right) \sim h\right| N\left(D_{2}\right)$. Let these equivalences be established by $\mathrm{f}_{1}: \mathrm{H}_{1}^{\prime}+\mathrm{H}_{2}^{\prime}$ and $\mathrm{f}_{2}: \mathrm{N}\left(\mathrm{D}_{1}\right)+\mathrm{N}\left(\mathrm{D}_{2}\right)$. By the homogeneity of 
the manifolds $\left(\mathrm{H}_{i}^{\prime}\right) *$ and $\mathrm{N}_{\left(\mathrm{D}_{1}\right) *,}$ ) $=1,2$, one may assume that $\mathrm{f}_{1}\left(\mathrm{H}_{1}^{\prime} \cap \mathrm{N}\left(\mathrm{D}_{1}\right)\right)=$ $\mathrm{f}_{2}\left(\mathrm{H}_{2}^{\prime} \cap \mathrm{N}\left(\mathrm{D}_{2}\right)\right)$. The equivalence $\mathrm{h} / \mathrm{H}_{1} \sim \mathrm{h} / \mathrm{H}_{2}$ is established by $\mathrm{f}: \mathrm{H}_{1}+\mathrm{H}_{2}$ which is reconstructed from the homeonorphism $\mathrm{f}_{1}$ and $\mathrm{f}_{2}$.

In order to modify the above argument so that $\mathrm{f} / \partial \mathrm{H}_{1}$ is the identity on $\mathrm{H}_{1} \cap \mathrm{H}_{2}$ we need only to be able to choose $D_{1} \subset H_{1}$ and $D_{2} \subset H_{2}$ so that $\partial D_{1}=\partial D_{2}$. The remainder of this proof consists in showing that $D_{1}$ and $D_{2}$ can be so chosen.

Consider the disk $D_{i}$ properly embedded in $H_{i}$ and cutting $F\left(h / H_{i}\right)$ as provided by Nelson [4; Theorem B]. Let $p$ denote the projection onto the orbit space $H_{1}^{*}$. Then $p\left(D_{i}\right)$ is a disk properly embedded in $H_{i}^{*} \cdot \partial p\left(D_{i}\right)=\alpha_{i} U \beta_{1}$, the union of two arcs, where $\alpha_{i} \subset p\left(F\left(h H_{i}\right)\right)$ and $\beta_{i} \subset p\left(\partial H_{i}\right) . D_{2}$ can be isotoped onto $D_{1}$ relative to $F$ if and only if $\alpha_{2}$ can be isotoped onto $\alpha_{1}$ relative to $\mathrm{p}\left(\mathrm{F}\left(\mathrm{h} / \mathrm{H}_{2}\right)\right)$ and $\beta_{2}$ can be isotoped onto $B_{1}$ relative to $\mathrm{p}\left(\partial \mathrm{H}_{2}\right)$. We can find such $\mathrm{D}_{1}$ and $D_{2}$ by the following procedure.

Fix $D_{1}$ and regard $\partial D_{1}$ as a fixed loop on $\partial H_{2}$. Employ Nelson [4; Theorem B] to find $\mathrm{D}_{2} \subset \mathrm{H}_{2}$. If $\alpha_{2}$ and $\beta_{2}$ can be isotoped relative to $\mathrm{p}\left(\mathrm{F}\left(\mathrm{h} / \mathrm{H}_{2}\right)\right.$ ) onto $\alpha_{1}$ and $\beta_{1}$, respectively, then we are done. If not, cut $\mathrm{H}_{2}$ along $\mathrm{D}_{2}$ to get a handlebody $\mathrm{H}_{2}^{\prime}=\mathrm{cl}\left(\mathrm{H}_{2}-\mathrm{N}\left(\mathrm{D}_{2}\right)\right)$ of lower genus. $\partial \mathrm{D}_{1}$ may still be regarded as a fixed loop on $\partial \mathrm{H}_{2}^{\prime}$. Note that with this cut the bordered surfaces $\mathrm{p}\left(\mathrm{F} \cap \mathrm{H}_{2}^{\prime}\right)$ and $\mathrm{p}\left(\partial \mathrm{H}_{2}^{\prime}\right)$ which make up $\partial\left(\mathrm{H}_{2}^{\prime}\right)^{*}$ have lower genera than $\mathrm{p}\left(\mathrm{F} \cap \mathrm{H}_{2}\right)$ and $\mathrm{p}\left(\partial \mathrm{H}_{2}\right)$, respectively. Now apply Nelson [4, Theorem B] again to $\mathrm{H}_{2}^{\prime}$ taking additional care that $\mathrm{D}_{2}^{\prime} \mathrm{misses}$ the "scars" of previous cuts (so that $\mathrm{D}_{2}^{\prime}$ may be regarded as properly embedded in $\mathrm{H}_{2}$ ). This can be done because of the homogeneity of $\partial\left(\mathrm{H}_{2}^{\prime}\right) *$. Repetition of this procedure must eventually yeild the desired disks $D_{1}$ and $D_{2}$ since the procedure eventually terminates with both $\mathrm{p}\left(\partial \mathrm{H}_{1}^{\prime}\right)$ and $\mathrm{p}\left(\mathrm{F} \quad \mathrm{H}_{2}^{\prime}\right)$ being disks.

\section{REFERENCES}

1. ROURKE, C.P. and SANDERSON, B.J., Introduction to Piecewise-Linear Topology, Springer-Verlag, Berlin, 1972.

2. BING, R.H., An Alternative Proof that 3-Manifolds can be Triangulated. Ann. of Math 69(1959), No. 2, 37-65.

3. NELSON, R.B., Some Fiber Preserving Involutions of Orientable 3-dimensional Handlebodies, Houston J. of Math. 9(1983), No. 2, 255-269.

4. NELSON, R.B., A Unique Decomposition of invulutions of Handlebodies, Proc. Amer. Math. Soc., 93(1985), No. 2, 358-362.

5. SEIFERT, H. and THRELFALL, W., Lehrbuch der Topologies, Chelsea Publishing Company, New York, 1947.

6. DOWNING, J.S., Decomposing Compact 3-manifolds into Honeomorphic Handlebodies, Proc. Amer. Math. Soc. 24(1970), 241-244.

7. KIM, P.K. and TOLLEFSON, J.L., PL Involutions of fibered 3-manifolds, Trans. Ame r. Math. Soc. 232 (1977), 221-237. 


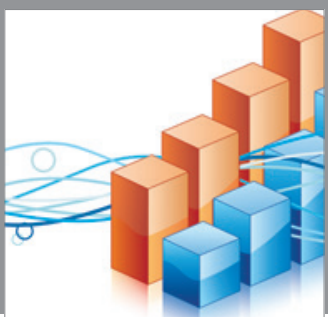

Advances in

Operations Research

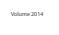

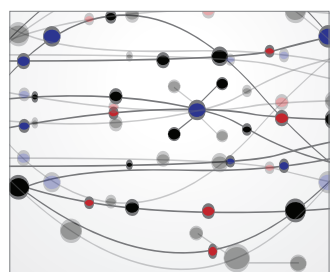

\section{The Scientific} World Journal
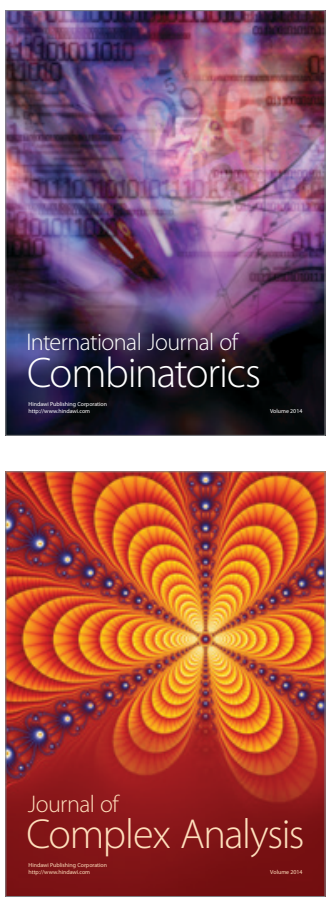

International Journal of

Mathematics and

Mathematical

Sciences
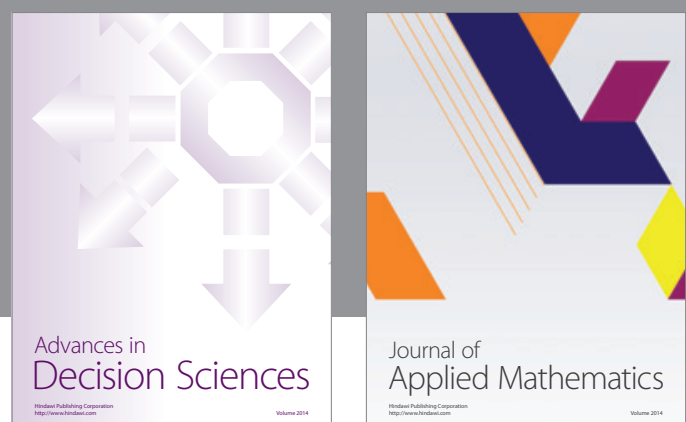

Journal of

Applied Mathematics
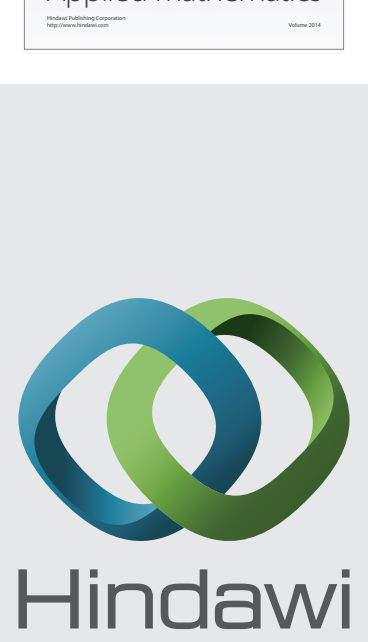

Submit your manuscripts at http://www.hindawi.com
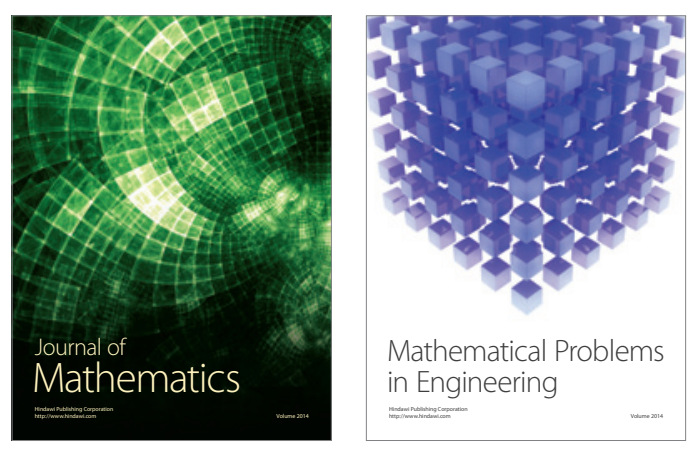

Mathematical Problems in Engineering
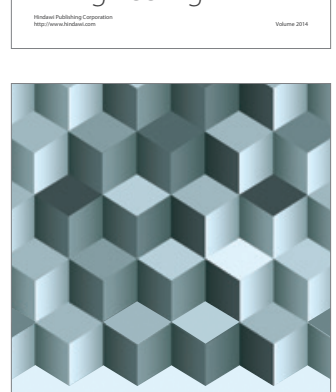

Journal of

Function Spaces
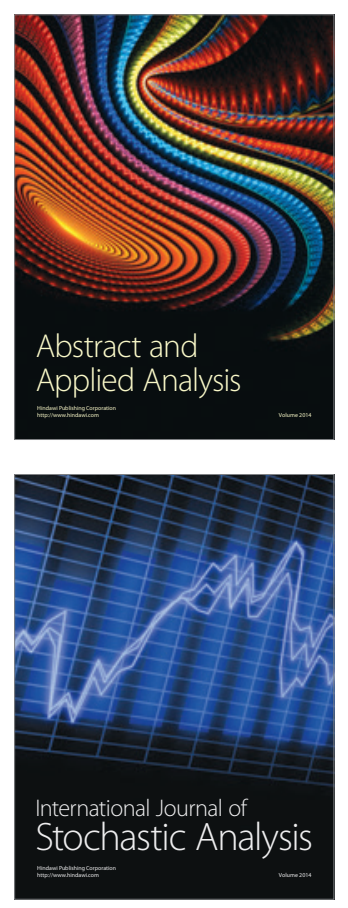

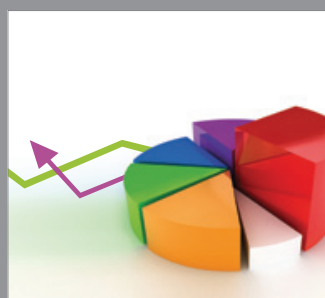

ournal of

Probability and Statistics

Promensencen
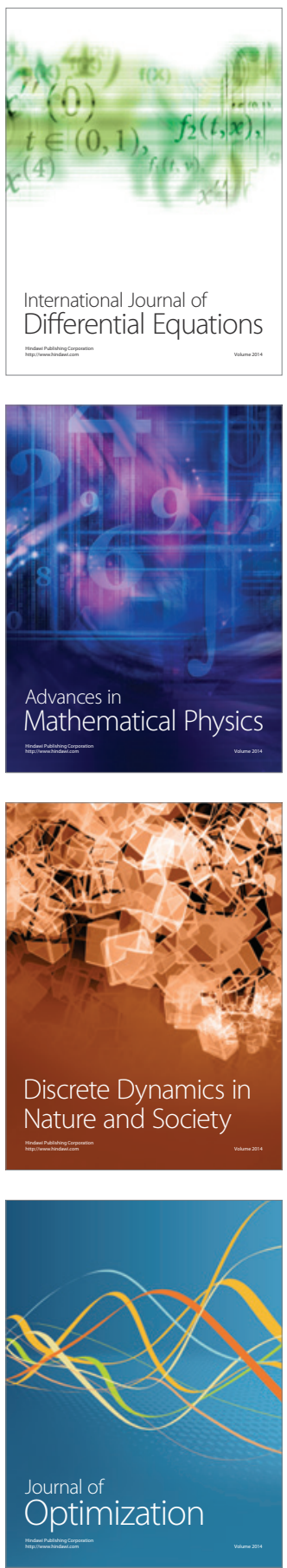